\title{
Der Bau des Löwenhofes im Kölner Rathause, 1541 und 1594.
}

Von

\section{Johannes Krudewig.}

Mit der Herstellung eines neuen Erdgeschossgewölbes im Kaufhause am Altermarkt unter dem Steinmetzmeister Tilman v. d. Urdenbach, die am 30. Januar $1540^{1}$ ) vom Rate beschlossen worden ist, haben die älteren, rein gotischen Bauten am Kölner Rathause ihren Abschluss gefunden.

Schon im nächsten Jahre setzte aber eine neue, glänzende Bauperiode ein, die bereits ganz dem Renaissancestil angehört. Dor wesentliche Zweck dies er Neubauten war, das alte Rathaus und das Kaufhaus am Altermarkt besser miteinander $z u$ verbinden und letzteres, mit Ausschluss der neuen Erdgeschosshalle, mehr den Rathauszwecken nutzbar zu machen ${ }^{2}$ ).

Der Rat deputierte am 4. März 1541 ,zome bouwe am raithuse: Beide herren burgermeistere zer zyt, Peter vann Krufftz, Herman Suyderman, Fredrich van Echt, Dierich Horner." ${ }^{63}$ ) Dieser Ausschuss hat nunmehr eine wesentliche Vervollkommnung des Rathausbaues durchführen lassen.

Nachdem nian den Eingang vom Altermarkt her dem Verkehr entsprechend umgestaltet, die alte, vom Hofe nach der Prophetenkammer führende hölzerne

1) Köln, Stadt-Archiv, Ratsprotok. 10, f. $176 \mathrm{~b}$.

2) Hans Vogts, Das Kolner Rathaus: Jahrb. des Koln. Geschichtsvereins, II (1913), S. 19.

8) Koln, Stadt-Archiv, Ratsprot. 10, f. 253 b, d. d. 1541 Mărz 4. 
Treppe niedergelegt und an deren Stelle eine prachtvolle, überwölbte Steintreppe errichtet hatte, erhielt auch der Raum zwischen dem Turm und den Rentkammern, der eine "schmutzige, unflätige“ Kloake gebildet hatte, seine endgültige Ausgestaltung ${ }^{1}$ ): In gleicher Ebene mit dem Rathausplatze baute man zur leichteren Verbindung der beiden Rentkammern einen auf starken Gewölben ruhenden Umgang; dieser umgab im unregelmässigen Viereck einen ein Stockwerk tiefer liegenden Hofraum, den nachmals sogenannten Löw en h of, welcher geebnet und mit Steinplatten belegt wurde und später als Hofraum des Hauses ,Zum Hirz" diente. Der ganze Bau wurde in dem eben in Aufnahme gekommenen Renaissancestil - „up antix", wie die noch vorhandene, eingehende Baurechnung ${ }^{2}$ ) sich ausdrückt, ausgeführt. Diese Rechnung des städtischen Rentmeisters ${ }^{3}$ ) iiber die Steinhauer- und Bauarbeiten des Steinmetzmeisters Laurenz gibt genauen Autschluss über die bei dem Baue zur Ausführung gebrachten Steinmetzarbeiten: die Bogensteine, die Wappen mit Laubwerk, das Geländer rund um das Werk, das ausgezeichnete Bildwerk "up antix" mit Laubgewinden, darauf man liegen soll, die welschen Bögen mit den welschen

1) [Ennen] in der Kolnischen Zeitung vom 11. September 1864, I, Nr. 253, und in Baudri, Organ für christl. Kunst, XIV (1864 November 15), S. $256 \mathrm{f}$, , sowio in der Belletristischen Beilage zu den Kölnischen Blattern, 1866 September 2, Nr. 23 ; hier und im Folgenden. Vgl. auch: Ennen in der Zeitschrift für bildende Kunst XI (1876), S. 282.

2) Koln, Stadt-Archiv, Rechn. Nr. 504 a. Diese Rechnung benutzte im Jahre 1864 Enden an den oben angegebenen Stellen ohne Zitat; sio war seitdem verschollen, bis ich sie 1901 in ungeordneten Bestănden des Kolner Stadtarchivs wiederfand. Siehe den vollstandigen Wortlaut unten foligend.

3) Wobl Al off Rink, wie folgende Eintragungen in dem Ämterverzeichnis der Ratslisten im Kolner Stadt-Archiv vermuten lassen: „1541 Jacob Rottkirchen, † 1541 im Sept., et in locum ipsius surrogatus est Goswin van Lomersheim Veneris ultima Septembris praesentis anni. 1541 ber Adolff Rynck vur ein jair; obiit in praesenti anno ipso die, ind in desselben stat ist gekoren worden Heinrich Broich Lune ultima Octobris praesentis anni. 1541 her Aloff Rinck vur ein jair ist er continuiert worden." 
„eppelen“ über der Pissine, die Schildbögen Fufssteine, Pfeiler, Kapitäle u. a. m.'). Die Baukosten beliefen sich auf 575 Gulden, 8 Albus und 7 Heller, oder, in Radergeld umgerechnet, auf 383 Gulden, 14 Albus $\left.{ }^{2}\right)$.

Meister Laurenz erhielt für den Bau 1542 vom Rat ein Geschenk von 50 Goldgulden. Er ist zweifellos mit dem Bildhauer Laurenz von Kronenburg identisch, der 1539 als Domwerkmeister, später als Baumeister der ehemaligen St. Jakobskirche, 1542 als Amtsmeister und Ratsumlauf erwähıt wird und 1547 aus dem Ratsdienst entlassen wurde").

Sein Bau zeigt im Untergeschoss unprofilierte Spitzbogen von $2,63 \mathrm{~m}$ Spannweite, die denen der Altermarkthalle der Form nach ähnlich sind; darüber befinden sich jetzt an drei Seiten je drei schlanke Rundbogen, an zwei gegenüberliegenden Ecken zwei schmälere Bogen und an der vierten, östlichen Seite vier Rundbogen, im ganzen 15 Bogen. Ihre Anzahl entspricht zwar genau den Angaben der Baurechnung, aber diese 15 Bogen stammen nicht alle von dem ersten Bau, vielmehr umfasste dieser nur die Bogen an der Westseite (siehe die Abbildung). Als Brüstung oder Geländer dienen Relieffelder mit Kränzen, Medaillons und Wappen, die von Tritonen gehalten werden. „Diese Füllungen sind nicht sonderlich geschickt zusammengestellt und in ihren Mafsen etwas plump. Man erkennt an ihnen, sowie an den Verhältnissen der oberen Galerie, wie ungewohnt dem Meister die Arbeit im neuen Stil war, während die noch gotischen Bauten und Steinmetzarbeiten, z. B. die unteren Bogen, die eigenartigen oberen Gewölbe des Umganges mit ihren sich in Stabform durchschneidenden Gurtbögen die Renaissanceteile an Güte der Arbeit und der Erfindung

1) En $\dot{n} \theta \mathbf{n}$, a. a. 0 .

2) Siehe unten die Gesamtabrechnung. Auf welcher Unterlage die Angabe von 633 Gulden beruht, die sich bei Ennen an den oben S. 79 Anm. 1 angegebenen Stellen und hiernach auch bei Vogts (a. a. 0.) findet, ist nicht festzustellen.

8) Vogts a. a. 0. Vgl. Merlo: Kölnische Künster, Sp. 179, 180 


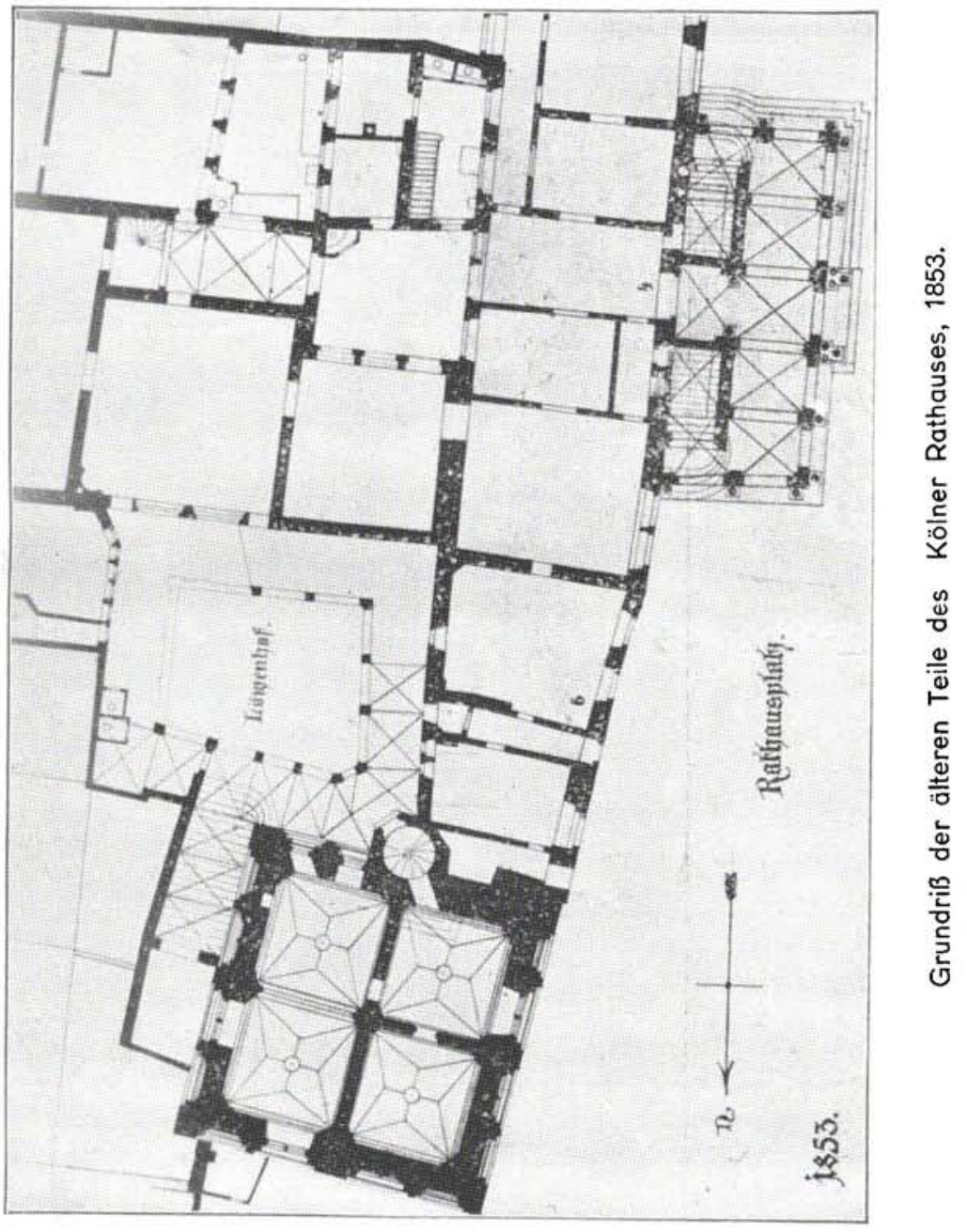


Brought to you by | New York University Bobst Library Technical Services Authenticated 


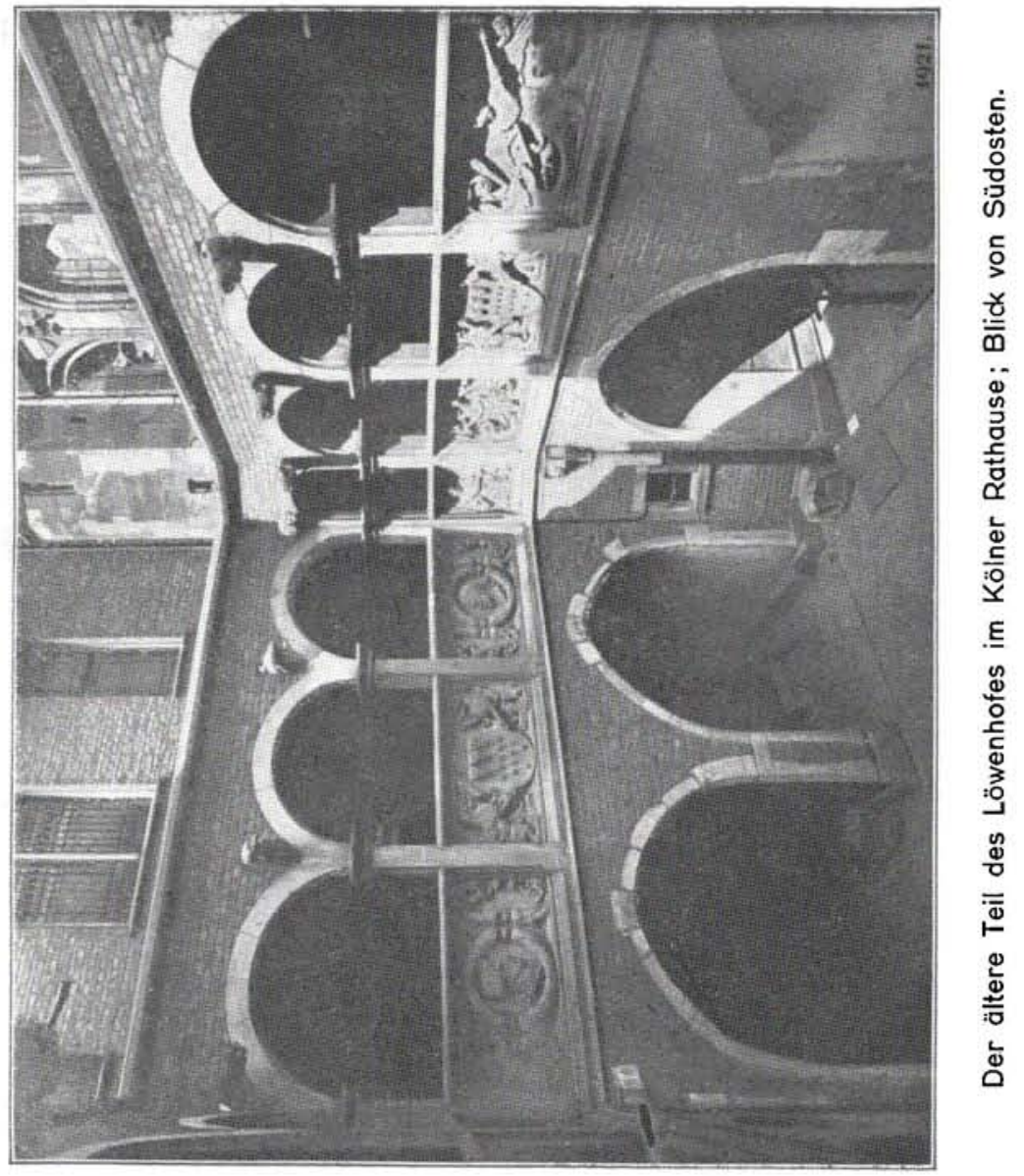


Brought to you by | New York University Bobst Library Technical Services Authenticated 
Der Bau des Lơweuhofes im Kolner Rathause, 1541 u. 1594.

übertreffen. Als Ganzes bietet der Löwenhof, besonders in Verbindung mit dem Turm, ein malerisches Bild." ${ }^{1)}$

Später diente der südliche Teil des unteren Bogenganges als Archivraum für die Rentkammer. ${ }^{1)}$

\section{Die Bau-Rechnung selbst lautet folgendermalsen:}

f. 1. Dyt yst dio Rechenschafft van dem Bouwo tuyschen beydenn Renthkaemeren, wes ych verlacht und betzalt hayn Meyster Laurens vur arbeydt und ethliche gereytschafft, und dat ander ist uff der Renthkaemeren durch dio herren Renthmeysteren betzalt anno 1541.

f. $1 \mathrm{~b}$, unbeschrieben.

f. 2. Item hayt Meyster Laurens gerechent per synen tzedell gehouwen unnd verdeynt deysse nageschreven parcelen anno 41.

Item gehouwen van myner herren steyn zu dem oyrsten $6 \overrightarrow{6} 6$ foyss schenckell den foyss vur 16 baller, facit zusamen 37 gulden 13 albus 4 haller

Itom 296 foyss groesser schenckell den foyss $3 \beta$ facit 18 gulden 12 albus

Item 128 foyss und 1 fyrdell pilerboegen den foyss $5 \beta$ facit 13 gulden 8 albus 8 haller I em 146 foes dach syms den foyss vur 20 haller facit 10 gulden 3 albus 4 haller Item 66 fooss plancken yederen foyss 8 haller facit 1 gulden 20 albus

Item 40 foess gewonden schenckell den foyss 32 baller facit

$\begin{array}{ccc}4 \text { gulden } & 10 \text { albus } & 8 \text { haller } \\ \text { Summa } & 85 \text { gulden } 19 \text { albus } & 10 \text { haller }\end{array}$

f. $2 \mathrm{~b}$. Item 60 foess durch sluyffen schytbogen den foyss 32 hallor facit

Item 15 floy sstoyn in dio schytboegen dat stuck 6 albus facit

6 gld. 16 alb.

Item 15 wapen myt louyffwerck und 15 floysssteyn in die crux tzosamen vur

Item dat gelens rondt umb dat werck 52 stuck 3 gld. 18 alb. 16 gld. ist uysgehauwen myt byldwerck up antyx mydt louffwerck, daer man up lygen sall, tzo samen gerechent vur

Item der welsche boege myt 3 welschen eppellen boeven der pyssynen vur 70 gld. 4 gld. 12 alb. Summa facit 100 gld. 22 alb.

1) Vogts, a. a. 0 .

Jahubuch des Kinhuischen Geschichtsvercins 5 . 
f. 3. Item noch 3 crax flosssteyn und 4 schyltboegen in die widt gewulff zu samen

Item 2 poesementen van hardem steyn dat stuck

$6 \mathrm{mr}$. facit

Item 2 pyler van hardem steyn gebrochen noch kosten

Itom 68 klaeger gehouwen dat stuck 15 haller facit

Itom 2 halff kaptheyll tuyschen dio poesementen gehouwen vur

Item noch layssen houwen van hardem steyn

11 gargell, dae dat wasser durch louff, vur

Item noch gehouwen 15 durchlouffenden flosssteyn, kosten

Item noch hait meyster Laurens verlacht an kalck und ander parcelen

3 gld.

3 gld.

4 gld.

3 gld. 12 alb.

1 gld.

11 gld.

8 gld.

9 gld. 6 alb. Summa facit 42 gld. 12 alb.

6 baller.

f. $3 \mathrm{~b}$. Item betzalt meyster Laurens, dat bey und dry gesellen zu samen gearbeydt hadt van dem 9. dach Mertz, wardt hey lest van der renthkameren betzalt, und eho ych ome gelt gaff, hat hey myt den 3 gesellen zu samen verdeynt myt 2 opperknechten, facit betzalt

35 gld. 8 alb.

Item noch betzalt 2 steynmetz, 1 opperknecht 5 dach facit

3 gld. 4 alb.

Item betzalt dem wechmecher, die goss zu machen vau hern Wasserfass huyss byss vur Myrwelich, zu samen verdeyndt

4 gld. 14 alb.

Summa facit 43 gld. 2 alb.

f. 4. Item betzalt meyster (Johann) [durchstrichen] Heynrich [Korrektur von anderer Hand] Fudenckar vur die blawe steyn zu dem estrich; sall haeven 50 gulden, der hadt hey up der renthkaemeren ouch 25 entfangen, und ych hain ene daer up betzalt. 10 gld.

Item noch betzalt moyster Heynrich Fuydenkars
15 gld.
de rest vur de blae steyn
solutum anno 42 in februario.
Item verdynckt meister Laurens vort dat werck
(5) dem raetzkeller und vur der rentkameren reyde
tzo machen up lerssaevent und sall eme daor van
betzalen up myner heren gereitzschafft vur synen
abeitzloen

Summa facit 65 gld. 
f. 4 b. Item in Mayo hait gelewert Noldon Jan

5000 steyn dat $1 \mathrm{~m}$ vur $9 \mathrm{mr}$. facit

Item betzalt prima Julij vur 2 foeren

lialx myt dem beslan gelde 12 albus facit

Item betzalt Nolden Jan den 3. Augusti

4000 steyn zu $9 \mathrm{mr}$., foerloyn $6 \mathrm{mr}$.

Item den 8 . Septembris 1 focr kalx myt

dem beslain 6 albus facit

Item den 17. Septembris 2 foeren kalx

myt beslaengelde 12 albus facit

11 gld. 6 alb.

6 gld. $3 \frac{1}{2}$ alb.

10 gld. 12 alb.

3 gld. 1 alb. 9 hall.

6 gld. $3 / 2$ alb.
Summa 37 gld. 2 alb. 9 hall.

f. 5. Item in Octobri, als ych zu Aich was, hain ych

nae betzalt 2 foeren balx myt dem ungelde facit

6 gld. $3 \frac{1}{2}$ alb.

Item noch in Octobrij betzaldt Heynssen Jan vur

3500 steyn zu 9 mar. facit

7 gld. 21 alb.

Item betzalt noch, dat Baitholmeus verlacht hadde,

$1 \mathrm{~m}$ und eyn derdendeyll steyn, facit mit dem voerloyn

betzalt

3 gld. $9 \frac{1}{2}$ alb.

Summa 17 gld. 10 alb.

f. 5 b. Item betzalt meyster Laureus den dachloyn anno 41 die weche var pynxsten angehaeven, die eyrste weche 4 man, yeder 6 dage, den dacb 6 albus, und eynen opperknecht 6 dach, yeder dach vier albus summa betzalt

Item die eyrste weche va pyoxsten 3 man und eynen opperknecht 2 dach facit betzalt

Item die 2. weche 3 man, eynen opperknecht, yoder 5 dach facit

Item dio 4 . woche 4 man und eynen opperauecht, yeder hadt 4 dach, facit

Item die 3. weche 3 man 5 dach, noch eynen

2 dach, eynen opperknecht 5 dach facit

7 gld.

1 gld. 20 alb.

4 gld. 14 alb.

4 gld. 16 alb.

5 gld. 2 alb.

Summa 23 gld. 4 alb.

f. 6 . Item die 5. weche, 13. Julij, 4 man, eyneu opperkuecht, der hat 6 dach, facit

Item betzalt vur 11 kaeren sandz zu 2 albus facit

7 gld.

Item die 6. weche 3 man 6 dach, noch eyner hayt vier dago, ein opperkuechte 6 dach summa botzalt

Item dio 7. und 8. weche, den lesten Julij, 5 man haudt zusamen verdeynt die 2 wechen $33 \frac{1}{2}$ mark, eyn opperkuecht 8 dach, facit 32 alb.

Item die 9. weche 5 man 6 dach, eynen opperlnecht seefs dach, summa facit

\begin{tabular}{rr}
8 gld. & 12 alb. \\
\hline Summa 32 gld. & 15 alb.
\end{tabular}


f. 6 b. Item die 10 . weche 5 man, yeder 5 dage, eynen opperknecht 5 dago, facit

Item die 11. weche 5 man, eynen opperknecht, yeder 5 dach facit zusamen betzalt

Item die 12. weche 4 man, eynen opporknecht, yoder vunff dach facit zusamen

Item die 13. woche 4 man, eynen opperknecht, edor 5 dach facit

Item die 14. weche 4 man und 2 opperknecht, yeder 5 dach facit zusamen

Item dis 15. weche 4 man, yeder 5 dach, no[ch]

7 gld. 2 alb.

6 gld. 2 alb.

5 gld. 20 alb.

5 gld. 20 alb.

6 gld. 16 alb. einen 1 dach, 2 opperknecht, yeder 5 dach summa facit 6 gld. 22 alb.

Summa 38 gld. 10 alb.

f. 7. Item die 16. weche 4 man, yeder 5 dach, noch 1 hadt $4 \frac{1}{2}$ dach, 2 opperknecht haben $9 \frac{1}{2}$ dach summa facit betzait

Item die 17. weche, 5 man, yeder 5 dach, 2 opperknecht, yeder 5 dach, summa facit

Item die 18. weche, 5 man, yoder 6 dach, eynen opperknecht 6 dacb, noch eynen $31 / 2$, summa facit

Item die 19. weche betzalt per Bartholomeum vur loen

Item die 20. weche hayt ouch Bartholomeys betzalt

7 gld. 17 alb.

7 gld. 22 alb.

9 gld. 2 alb.

6 gld. 14 alb. van niynent wegen

5 gld. 20 alb.

Summa 37 gld. 3 alb.

f. $7 \mathrm{~b}$. Item die eynundzwensigste weche, den 29 . Octobris betzalt 4 man und 1 opperknecht, yeder vunff dach, facit zusamen

5 gld. 20 alb.

Item die 22. weche, 4 man, einen opperlsnecht, eder $4 \frac{1}{2}$ dach, facit

Item die 23. weche, 4 man, 1 opperknecht, yederem 4 dach facit

5 gld. 6 alb.

Item die 24. weche, 4 man, 1 opperknecht, yederem

6 dach facit

4 gld. 16 alb.

Item die 25. weche, 4 man, 1 opperknecht, eder 3 dach, facit

7 gld.

3 gld. 12 alb. Summa 26 gld. 6 alb.

f. 8. Item die 26. weche, 4 man und eynen opperknecht, yeder vunff dach, facit

5 gld. 20 alb.

Item die 27. weche, 4 mann, eynen opporknecht, yederem 4 dach facit

4 gld. 16 alb. 
[Von anderer gröberen Hand, wie auf f. 4:]

Item de 28. weche 4 man, eynen opperknecht,

eder 6 dach facit

7 gld.

Item de 29. weche gynck uyls up Kerlsaevent

4 man, eder 5 dach facit

5 gld.

Item betzalt vur qwestfloek buyden eyn aem

beyrfs den gesellen tzo samen

3 gld. $91 / 2$ alb.

Summa facit 25 gld. $21 \frac{1}{2}$ alb.

Summa summarum alles, wes ich an den bouwe verlacht und betzalt hain nae luydo

deser parsolen in diesem boech, facit 575 gld. 8 alb. 7 hall.

facit tzo radergelt, den raderalbus tzo 18 heller, als ich betzalt hain, und tzo 24 raderalbus vur den gulden, facit 383 gulden, 14 raderalbus, tzo 24 raderalbus vur den gulden.

Eine eingehende Ortsbesichtigung hat ergeben, dass sich diese Rechnnng nicht nur auf den Bau des Löwenhofes, sondern auch anderer Teile des Rathauses, besonders des Durchganges zum Muschelsaal, bezieht. Wenn man dieses ins Auge fasst, so kommt man auch mit der Zählung der 15 Gewölbe oder Bögen und ihrer Wappen (Schlufssteine) aus; denn die drei Gewölbe des genannten Durchganges sind in der Anlage dieselben, wie die alten im westlichen 'Teile des Löwenhofes.

Rund fünfzig Jahre später, im Jahre 15y4, mussten an dem Bauwerk weitere Bauarbeiten vorgenommen werden, die Unkosten in Höhe von 600 Gulden verursachten ${ }^{1}$ ). Welcher Art diese Arbeiten im einzelnen gewesen sind, lässt sich nicht mit Sicherheit sagen. Die Ratsprotokolle äussern sich darüber folgendermafsen :

$$
\text { Mercurij, 5. Jannarij ao. [15]94. }
$$

Bau an der Guidestags Rentcammer.

Der her rentmeister Suderman hat mundtlich vorgedragen, das die herren rentmeister und beysitzer der Godestagsrentcammer cynen notigen bau neben der rentcammeren besichtigt und den ganck so vermauert; und als mit vermeldet, dass der bau mit 600 daler verdingt

1) B a udri: Organ f. christl. Kunst, XIV (1864), S. 257. 
und verfertigt kan werden, hat eyn erbarer rheidt sollichs beliebet ${ }^{1}$ ).

Man darf annehmen, dass damals der östliche Teil des oberen Arkadenganges gebaut worden ist. In das mittlere Relieffeld wurde eine der damals in Köln sehr beliebten Darstellungen des Löwenkanıfes des sagenhaften Bürgermeisters Gryn hineingesetzt; das war die Veranlassung zu der späteren Benennnng „Löwenhof" "2). Das Relief des Löwenkampfes trägt nämlich die Jahreszahl 1594 und in den beiden obern Ecken die Wappen der regierenden Bürgermeister dieses Jahres: Hildebrand Suderman und Gerhard Angelmacher. $\mathrm{Es}$ ist jetzt am Gereonswall in die Aussenmauer des Schnïtgen - Museums eingelassen und im Löwenhof durch eine Kopie ersetzt, die, von der weggelassenen Quadrierung des Hintergrundes abgesehen, das Original getreu wiedergibt.

Im Jahre 1531 erwähnt Weinsberg das alte Portal des Rathauses als "das Portal boven den peilern". Auf einem der Pfeiler befand sich eine antike, in Stein gehauene Darstellung des Kampfes des Herkules mit dem nemëischen Löwen; diese wird wohl beim Volke zu der Sage von dem Kampf eines kölnischen Bürgermeisters Hermann Gryn mit cinem Löwen Veranlassung gegeben haben. Ein die Begebenheit darstellender Kupferstich von 1571 trägt folgende Unterschrift: „1262 . . und des tzo eyner ewiger gedechnisse is eyne steinen gehauwen bildo Herman gryns mit dem leuwen op einen van den pilren gesatzt vur dem Raithuse und auch gemaelt in der Herrn clagekamer op dem Raithuys."

1) Koln, Stadt-Arch., Rpr. 44, f. 103.

2) Vgl. die eingehende Bearbeit ing „Der Lowenkampf des Bürgermeisters Grin" von Joseph Klinkenberg in dem Kolver Heft der Mitteilungen des Rbeinischen Vercins für Denkmalpflege und Heimatschutz (V 1, 15. März 1911). 